\section{Misplacement of PICCs Following Power Injected CT Contrast}

\section{A Quantitative Descriptive Review}

$\mathrm{DOI}$

https://doi.org/10.38192/1.6.3.pnauk.wcc20. ab3

Epub: 11.10 .2020

Abstract presented at Philippine Nurses UK World Café Convention 24 October 2020

Keywords: vascular access devices,

Background: Peripherally inserted central catheters (PICCs) may be used to administer contrast injection during CT (computed tomography) scans to improve diagnostic accuracy. This is usually done with the use of a power injector. Research has shown that misplacement of catheters following contrast injection can occur.

Aim: We aim to study appropriately positioned pre scan PICC's that malposition following CT injection of contrast media, evaluate if tip location or right or left insertion plays a part in the displacement of PICC's post CT, and to ensure persons involved are aware of the risks and the safety checks required following such procedures.

Methods: Quantitative method was used, gathering information from the team's insertion database. It reviewed 2045 records of contrast injectable PICCs between 1st January 2015 and 30 April 2020. Information on misplaced PICCs following CT injection was collected in retrospect by reviewing the Vascular Access Team database. Power Injectable 4Fr and 5Fr PICCs that were inserted between 1st January 2015 and 30 April 2020 were included. This included PICCs that were confirmed to be okay for use after confirmation of tip position.

Basing on the comments on the database, entries with the words such as misplaced, CT and coiled (curled/loop/flipped) were included. This data was double checked with online chest radiograph images to confirm evidence of malposition following contrast injected CT Scan.

Results: A total of $21(1 \%)$ PICCs were reported to have been misplaced following contrast injection between 2015 and 2020. After contrast CT injection, the tip misplaced to the following locations, 7 (33\%) in Right Internal Jugular vein, 6 $(29 \%)$ in the left and $4(20 \%)$ in the right brachiocephalic veins, $2(10 \%)$ in the right subclavian vein, $1(5 \%)$ in the Left Internal Jugular vein and $1(5 \%)$ coiled in SVC (superior vena cava). Repeat x-rays were reviewed and 19 (90\%) PICCs showed evidence of self-direction. A total of 8 (38\%) PICCs demonstrated self-direction on repeat chest x-ray at 24 hours and $11(52 \%)$ at more than 24 hours (Mean $=64.4$ hours).

Conclusion: Analysis of the data indicate that $1 \%$ of appropriately sited PICCs malposition following contrast CT injection and that the catheter is able to self-direct back to its original position in 24-72 hours without any other intervention. However in some cases, it can take up to 72 hours for it to self-direct. There is a need to check PICC tip position following CT contrast injection as there is a risk of malposition. Further research is needed to explore other factors that could influence the rate of self-direction and complications of prolonged catheter malposition.

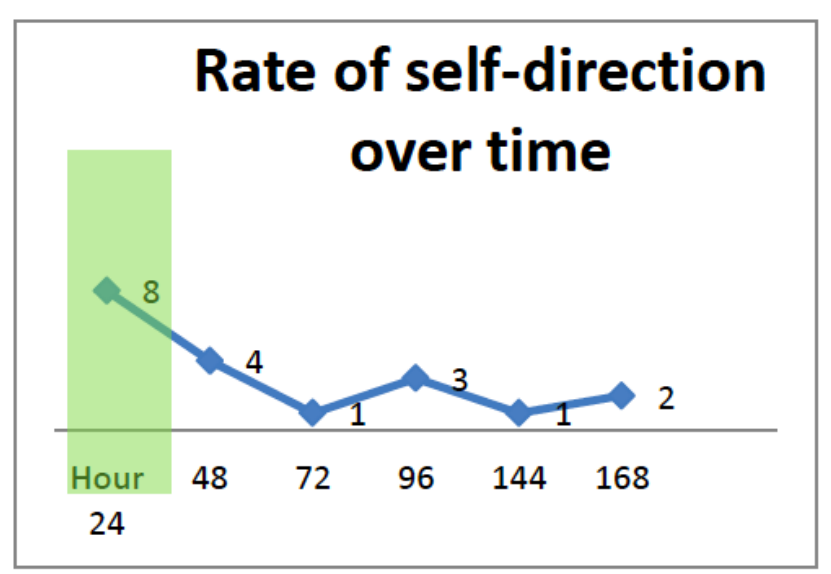

Funding: Not Applicable

Ethics: Not Applicable Declaration of Interest: Nothing to disclose 